\title{
Is postural tachycardia syndrome an autoimmune disorder? And other updates on recent autonomic research
}

\author{
Mitchell G. Miglis ${ }^{1,2} \cdot$ Srikanth Muppidi ${ }^{1}$
}

Received: 25 December 2019 / Accepted: 26 December 2019 / Published online: 14 January 2020

(c) Springer-Verlag GmbH Germany, part of Springer Nature 2020

\section{Is postural tachycardia syndrome an autoimmune disorder?}

Whether autoimmunity is involved in the pathophysiology of postural tachycardia syndrome (POTS) is a controversial and still undefined topic. When autoantibodies against ganglionic nicotinic acetylcholine receptors were first identified 20 years ago, it became clear that high titers were associated with severe autonomic failure including orthostatic hypotension, tonic pupils, and gastrointestinal and bladder abnormalities, a syndrome now referred to as autonomic autoimmune ganglionopathy (AAG). However, some patients with POTS also tested positive with low titers [13]. Autonomic experts now agree that positivity for ganglionic nicotinic antibodies at low titer (i.e., below $0.2 \mathrm{nmol} / \mathrm{L}$ ) has no clinical relevance for POTS, as these can be detected in up to $5 \%$ of healthy controls $[9,12,14]$.

More recently, three groups (one at the University of Oklahoma, one at Lund University in Sweden, and one at University of Toledo in Ohio) have reported elevated antibodies against different G-coupled protein receptors (GCPRs) in patients with POTS [3, 5, 7, 15]. These studies found that, compared to controls, the vast majority of patients with POTS had serum antibodies against $\alpha$-adrenergic and $\beta$-adrenergic receptors, and various subtypes of muscarinic, and angiotensin II receptors. These studies, consistently small (no more than 20 patients even in spite of the fact that POTS is highly prevalent in the general population), are not devoid of methodological limitations and difficulties when interpreting the results. Indeed, the most important criticism is the lack of correlation between the function of these autoantibodies and the phenotype

Mitchell G. Miglis

muppidis@stanford.edu

Stanford Medical Center, Palo Alto, CA, USA

2 Stanford Neurosciences Health Center, 213 Quarry Road, 2nd Floor, Palo Alto, CA 94304, USA of POTS. If these autoantibodies activated ubiquitous $\alpha 1$-adrenergic receptors throughout the autonomic system in patients with POTS, patients should suffer not only from tachycardia, which is the defining finding of POTS, but also hypertension, piloerection and urinary retention, among other manifestations. And so on with the other identified antibodies against $\beta$-adrenergic, muscarinic, and angiotensin antibodies. From a physiology point of view, it would be certainly challenging to imagine what would happen in a patient in whom all these receptors were activated and/or inhibited at the same time by these putative autoantibodies. The group at the University of Oklahoma also found the same antibodies in multiple, disparate disorders (e.g., orthostatic hypotension, atrial fibrillation, and primary aldosteronism $[4,6$, 16]) suggesting that their findings do not appear to be disease-specific. Others argue that while these autoantibodies may not have a direct pathogenic role in POTS, they could reflect: (1) some degree of immune dysregulation given that GPRCs play a role in regulating the innate immune system [11]; (2) an immune response to tissue injury; or (3) some type of physiological regulatory response to cardiovascular stress. Answering these questions is relevant for therapeutic reasons: disorders unequivocally caused by an autoimmunity (e.g., myasthenia gravis, Graves' disease) respond well to immunomodulatory treatments. In any case, at this time, adrenergic, muscarinic and angiotensin receptor antibodies have not been proven to be causative or useful in confirming a diagnosis of POTS.

An important limiting factor in exploring the mechanisms of autoimmunity in POTS has been the lack of an animal model. In their study recently published in the Journal of the American Heart Association, the University of Oklahoma group report the use of an animal model to investigate the role of adrenergic autoimmunity on the cardiovascular system [8]. Using a rabbit model previously employed in prior mechanistic cardiovascular studies, the authors tested the hypothesis that antibodies against the $\alpha 1$-adrenergic and $\beta 1$-adrenergic receptors may 
be involved in the pathophysiology of POTS. They immunized eight rabbits with peptides of the $\alpha 1$-adrenergic and $\beta 1$-adrenergic receptors with boosters at 2 and 4 weeks to elicit antibodies against these epitopes. They then performed cardiovascular autonomic testing in the rabbits, including ECG and intra-arterial blood pressure measurements on a tilt table, as well as phenylephrine (an $\alpha$-adrenergic agonist) and isoproterenol (a non-selective $\beta$-adrenergic agonist) infusions to examine the functional effects of the receptors. Each rabbit served as its own control.

At 6 weeks after immunization, heart rate increased by $17 \mathrm{bpm}$ on head-up tilt, compared to the rabbits' pre-immune baseline $(27.8 \pm 3.3 \%$ vs. $18.9 \pm 1.5 \% ; P<0.05)$. The heart rate increase was suppressed by custom-designed antibodyneutralizing peptidomimetic inhibitors that specifically target the antibodies and block their interaction with the receptors. There was no significant change in blood pressure on tilt testing. During the phenylephrine injection after immunization, heart rate responses were significantly attenuated, suggesting a negative allosteric effect of $\alpha 1$-adrenergic antibodies, while the heart rate was significantly increased with isoproterenol, suggesting a positive allosteric effect of the $\beta 1$-adrenergic antibodies. The authors found similar results using an in vitro cell-based bioassay, with rabbit sera obtained at the same timepoints as their in vivo analysis: before immunization, 6 weeks after immunization (before and after antibody-neutralizing peptide injection), and 30 weeks after immunization (after continued boost and antibody-neutralizing peptide treatments). They found an approximately 0.5 -fold increase in $\alpha 1$-adrenergic anitbody activity and a 10 -fold increase in $\beta 1$-adrenergic antibodies activity after immunization, and a reduction to pre-immune baseline activity in the presence of the antibody-neutralizing peptides. These findings suggest that these adrenergic antibodies in rabbits indeed induced tachycardia, which was suppressed by custom-made antibody-neutralizing peptides.

The article is certainly interesting although some limitations must be noted. The animal model used appears to be good to study baroreflex function; however, it tells us nothing about the other multiple manifestations that patients with POTS suffer from. While it is speculative to compare any animal model to the heterogeneous clinical realities of POTS in humans, nevertheless, the rabbit model appears to be the most reasonable methodological choice. It would have been interesting to see the effects of inoculating serum from a patient with POTS who tested positive for these antibodies in a healthy rabbit, or, at least, whether serum from a rabbit containing autoantibodies could elicit tachycardia in a healthy rabbit. Moreover, the authors used no negative control, which would have been useful to ensure that the increased in heart rate was not just due to the rabbits experiencing the stress of undergoing multiple inoculations over several weeks. There was no placebo arm to compare the effects antibody-neutralizing peptide activity.

Hence, the greater conceptual questions still remain: are these autoantibodies, which seem ubiquitous in the serum of POTS patients, a mechanistic cause of disease or rather a bystander effect of the disease process? If the autoantibodies are causative, should we expect evidence of autonomic failure due to tissue destruction at some point in the natural history of POTS, as we see in autoimmune autonomic ganglionopathy (AAG)? These are questions that are vigorously debated within the autonomic community and, as always, further studies are needed. Nonetheless, this study is an important step in the continued investigation of the role of autoimmunity in POTS. More importantly, it offers hope for potential customized immunological therapies, but only if these autoantibodies are confirmed to cause POTS.

\section{Skin biopsy for autonomic syndromes: time to look beyond nerve fiber density}

Skin biopsy has been used for assessment of small fiber dysfunction for many years and has become one of the standard diagnostic tool for assessment of small fiber neuropathy [10]. Many commercial laboratories provide intra-epidermal nerve fiber density analysis services, as it remains a simple, minimally invasive test to aid in the diagnosis of small fiber neuropathy. Additionally, several laboratories are in the process of offering $\alpha$-synuclein staining for diagnostic purposes in the setting of suspected Parkinson disease and other synucleinopathies [1]. Other measures of small fiber function such as quantitative sudomotor axon reflex testing (QSART), commonly used in autonomic laboratories, are a physiological correlate of small fiber function loss. In spite of these developments, skin biopsy and intra-epidermal nerve fiber density assessment remains a non-specific measure of peripheral autonomic fiber loss and cannot distinguish the loss of sympathetic, parasympathetic, or somatic fibers. This type of functional information would be valuable in assessment of autonomic syndromes with predominant sympathetic or parasympathetic fiber loss. Previous attempts to assess different autonomic fibers in skin samples were only partially successful.

In a recent publication in Scientific Reports, Donadio and colleagues used both immunofluorescent staining and confocal microscopy to comprehensively study and better inform the understanding of the autonomic innervation of the skin [2]. They obtained skin samples from 8 healthy asymptomatic participants ( 5 men, 3 women, mean age 45 years) without risk factors for neuropathy. The skin samples were obtained from the thigh $(15 \mathrm{~cm}$ above the patella) and the distal leg $(10 \mathrm{~cm}$ above the lateral malleolus). The researchers used a comprehensive set of markers to characterize 
autonomic fibers. To quantify adrenergic fibers, they used tyrosine hydroxylase $(\mathrm{TH})$ and dopamine- $\beta$-hydroxylase $(\mathrm{D} \beta \mathrm{H})$; to quantify cholinergic fibers, they used vesicular acetylcholine transporter (VAChT); they also used neuropeptide-Y (NPY), calcitonin gene-related peptide (CGRP), substance $\mathrm{P}(\mathrm{SP})$ and vasoactive intestinal peptide (VIP) as autonomic and neuropeptidergic markers. They stained different skin structures including sweat glands, skin arterioles, and arrector pili muscles and analyzed the samples with confocal microscopy.

The results showed that skin shows an incredibly complex autonomic innervation pattern. Skin arterioles were predominantly innervated by fibers staining positive for $\mathrm{TH}, \mathrm{D} \beta \mathrm{H}$ and NPY. Arrector pili muscles were innervated by fibers staining positive for TH, D $\beta H$, but not NPY. Sweat tubules were predominantly innervated by fibers staining for VAChT and VIP. Nerve fibers staining positive for VIP and CGRP without adrenergic or cholinergic staining were found in all structures at different rates of innervation. There was additional co-staining of cholinergic fibers in skin arterioles and arrector pili fibers. Moreover, sensory nerves were found in all the studied structures, suggesting a role in reflex regulatory mechanisms.

These finding support previously reported autonomic fiber innervation of various skin structures and, most importantly, demonstrates that advanced characterization of sensory and autonomic nerves can be readily performed, however currently limited to the research setting. With improved techniques and commercialization of methods, we hope that these findings might soon extend to clinical practice, and look forward to the possibility that skin biopsy can delineate not only the percentage of small fiber loss, but the characterization of adrenergic, cholinergic and somatic fiber involvement. The correlation of such pathological findings with clinical symptoms such as sweating abnormalities, neuropathic or vasomotor changes could be incredibly valuable, and might hopefully lead to better understanding of disease causation and therapy options in patients with small fiber and peripheral autonomic neuropathy.

Funding None.

\section{Compliance with ethical standards}

Conflict of interest None.

\section{References}

1. Donadio V (2019) Skin nerve alpha-synuclein deposits in Parkinson's disease and other synucleinopathies: a review. Clin Auton Res 29:577-585
2. Donadio V, Incensi A, Vacchiano V, Infante R, Magnani M, Liguori R (2019) The autonomic innervation of hairy skin in humans: an in vivo confocal study. Sci Rep 9:16982

3. Fedorowski A, Li H, Yu X, Koelsch KA, Harris VM, Liles C, Murphy TA, Quadri SMS, Scofield RH, Sutton R, Melander O, Kem DC (2017) Antiadrenergic autoimmunity in postural tachycardia syndrome. Europace 19:1211-1219

4. Galloway A, Li H, Vanderlinde-Wood M, Khan M, Benbrook A, Liles C, Zillner C, Rao V, Cunningham MW, Yu X, Kem DC (2015) Activating autoantibodies to the beta1/2-adrenergic and M2 muscarinic receptors associate with atrial tachyarrhythmias in patients with hyperthyroidism. Endocrine 49:457-463

5. Gunning WT 3rd, Kvale H, Kramer PM, Karabin BL, Grubb BP (2019) Postural orthostatic tachycardia syndrome is associated with elevated g-protein coupled receptor autoantibodies. J Am Heart Assoc 8:e013602

6. Kem DC, Li H, Velarde-Miranda C, Liles C, Vanderlinde-Wood M, Galloway A, Khan M, Zillner C, Benbrook A, Rao V, GomezSanchez CE, Cunningham MW, Yu X (2014) Autoimmune mechanisms activating the angiotensin AT1 receptor in 'primary' aldosteronism. J Clin Endocrinol Metab 99:1790-1797

7. Li H, Yu X, Liles C, Khan M, Vanderlinde-Wood M, Galloway A, Zillner C, Benbrook A, Reim S, Collier D, Hill MA, Raj SR, Okamoto LE, Cunningham MW, Aston CE, Kem DC (2014) Autoimmune basis for postural tachycardia syndrome. J Am Heart Assoc 3:e000755

8. Li H, Zhang G, Zhou L, Nuss Z, Beel M, Hines B, Murphy T, Liles J, Zhang L, Kem DC, Yu X (2019) Adrenergic autoantibodyinduced postural tachycardia syndrome in rabbits. J Am Heart Assoc 8:e013006

9. Li Y, Jammoul A, Mente K, Li J, Shields RW, Vernino S, RaeGrant A (2015) Clinical experience of seropositive ganglionic acetylcholine receptor antibody in a tertiary neurology referral center. Muscle Nerve 52:386-391

10. Myers MI, Peltier AC (2013) Uses of skin biopsy for sensory and autonomic nerve assessment. Curr Neurol Neurosci Rep 13:323

11. Sun J, Singh V, Kajino-Sakamoto R, Aballay A (2011) Neuronal GPCR controls innate immunity by regulating noncanonical unfolded protein response genes. Science 332:729-732

12. Vernino S, Hopkins S, Okamoto L, Black B, Dorminy C, Paranjape S, Raj S (2015) Prevalence of ganglionic AChR antibodies in postural tachycardia syndrome (POTS) (P1.276). Neurology 84:P1.276

13. Vernino S, Low PA, Fealey RD, Stewart JD, Farrugia G, Lennon VA (2000) Autoantibodies to ganglionic acetylcholine receptors in autoimmune autonomic neuropathies. N Engl J Med 343:847-855

14. Vernino S, Stiles LE (2018) Autoimmunity in postural orthostatic tachycardia syndrome: current understanding. Auton Neurosci Basic Clin 215:78-82

15. Yu X, Li H, Murphy TA, Nuss Z, Liles J, Liles C, Aston CE, Raj SR, Fedorowski A, Kem DC (2018) Angiotensin II type 1 receptor autoantibodies in postural tachycardia syndrome. J Am Heart Assoc 7:e008351

16. Yu X, Stavrakis S, Hill MA, Huang S, Reim S, Li H, Khan M, Hamlett S, Cunningham MW, Kem DC (2012) Autoantibody activation of beta-adrenergic and muscarinic receptors contributes to an "autoimmune" orthostatic hypotension. J Am Soc Hypertens JASH 6:40-47 